\title{
Case Report: The Combined Spinal and Caudal (CSC) Anesthetic-Is this the New Ideal Anesthetic in a Formerly Preterm, High-Risk Infant with Chronic Lung Disease Undergoing Infraumbilical Surgery?
}

\author{
Kelly Tseng, MD* and Chang Amber Liu, MD, MSc, FAAP \\ Department of Anesthesia, Critical Care \& Pain Medicine, Massachusetts General Hospital, Boston MA, USA
}

\begin{abstract}
Postoperative apnea is a well-recognized clinical entity commonly occurring in formerly preterm, high-risk infants. Spinal anesthesia has been demonstrated to be an attractive alternative to general anesthesia as it is associated with reduction of early risk of postoperative apnea in infants, hence it has become a valuable anesthetic technique in infraumbilical and lower extremity procedures, including inguinal herniorrhaphy. We present a case of a formerly preterm, high-risk infant with chronic lung disease undergoing bilateral inguinal herniorrhaphy and circumcision, in whom a successful spinal anesthetic was performed for bilateral inguinal herniorrhaphy and circumcision followed by a caudal anesthetic for postoperative pain control in a high-risk infant.
\end{abstract}

\section{Introduction}

Postoperative apnea in formerly preterm (gestational age $\leq 37$ weeks), high-risk (preterm infants whose post-conceptual age at the time of surgery is $<60$ weeks) infants is a well-recognized clinical entity that has been investigated by numerous reports since the late 1980's [1-4]. Although many factors have been implicated as the cause of postoperative apnea in this patient population, it is not certain whether it is a consequence of neurohumoral substances, postoperative pain, airway obstruction, diminished respiratory drive, hypothermia, anemia, residual general anesthetic effects, or other factors $[1,3]$. Nonetheless, literature has demonstrated that the incidence of postoperative apnea, which is often associated with postoperative desaturation and bradycardia, is higher in preterm, high-risk infants and this finding has thereby led to the routine practice of hospital admission of formerly preterm, high-risk infants for monitoring after receiving general anesthesia [1,3]. Endeavors to decrease the incidence of postoperative apnea after receiving a general anesthetic shifted the interest towards spinal anesthesia.

Spinal anesthesia, a safe anesthetic performed in children for over a hundred years, has been an attractive alternative to general anesthesia as it can circumvent inhalation or intravenous anesthetics and sedatives-agents that have been linked to apoptosis of neurons and oligodendrocytes, impaired hippocampal neurogenesis, and reduction in synapse number in animal models [5]. Thus in the 1990's-2000's, spinal anesthesia experienced its resurgence as evidence had demonstrat- ed that it was associated with postoperative apnea risk reduction compared to general anesthesia $[1,2]$. Over the last decade, spinal anesthesia has become a useful technique in infraumbilical, urologic, and lower limb surgeries in infants at a number of specialized pediatric institutions $[3,6,7]$. However, successful spinal anesthesia in infants depends on a multitude of factors including provider's skill, preoperative assessment, proper patient positioning during and after the spinal, and appropriate drug selection and dosing. Among these factors, spinal drug dosing is a contentious topic in research as the dosage of local anesthetics used for spinal anesthesia in children is not well determined [8]. In fact, many dose range finding studies have been conducted to determine the minimum local anesthetic dose (MLAD) and its corresponding response (duration of motor blockade). Additionally, recent studies have called into question the bodyweight-based dos-

\footnotetext{
*Corresponding author: Kelly Tseng, MD, Department of Anesthesia, Critical Care \& Pain Medicine, Massachusetts General Hospital, 55 Fruit Street, Gray-Bigelow 444, Boston, MA 02114, USA, Tel: (626)-478-8616

Accepted: October 14, 2020

Published online: October 16, 2020

Citation: Tseng K, Liu CA (2020) Case Report: The Combined Spinal and Caudal (CSC) Anesthetic-Is this the New Ideal Anesthetic in a Formerly Preterm, High-Risk Infant with Chronic Lung Disease Undergoing Infraumbilical Surgery?. J Clin Anesth Pain Manag 4(2):131-133
} 
Citation: Tseng K, Liu CA (2020) Case Report: The Combined Spinal and Caudal (CSC) Anesthetic-Is this the New Ideal Anesthetic in a Formerly Preterm, High-Risk Infant with Chronic Lung Disease Undergoing Infraumbilical Surgery?. J Clin Anesth Pain Manag 4(2):131-133

ing of local anesthetics for spinal due to the fact that there is a large variation in body weight among same age group infants; instead these researchers have proposed a new formula for spinal local anesthetic dosing based on age since spinal cord growth is proportional to age [9]. We report the administration of a combined awake spinal (bodyweight-based dosed) followed by a caudal anesthetic at the end of the surgery in a formerly preterm, high-risk infant with chronic lung disease who underwent bilateral open inguinal herniorrhaphy and circumcision.

\section{Case Description}

A former 28 0/7 week gestation male at 40 3/7 weeks postconceptional age was scheduled for bilateral inguinal hernia repair and circumcision. His past medical history and hospital course was notable for delivery via cesarean section due to non-reassuring fetal heart rates and breech presentation with oligohydramnios and preterm premature rupture of membranes at 23 weeks. He was admitted to the Massachusetts General Hospital NICU for evaluation and management of issues related to prematurity. Prior to birth, he was treated with latency antibiotics, two courses of betamethasone, and a magnesium bolus. At birth, he was non-vigorous with poor tone and cry. Interventions included active warming, stimulation, and bulb suctioning with progression to noninvasive positive pressure ventilation (NIPPV) and subsequent intubation. Apgar scores were $2,6,7$. He received surfactant on day of life (DOL) 0 , was extubated to NIPPV and started on caffeine on DOL 1 , and was weaned to continuous positive airway pressure (CPAP) on DOL 7 then to nasal cannula and ultimately to room air (RA) on DOL 72 . During that time he was also treated with diuretic therapy, as well as parenteral nutrition with advancement to enteral nutrition, phototherapy for hyperbilirubinemia, and blood transfusion for anemia, desaturation/apnea, and bradycardia. He continued to remain stable throughout admission and was scheduled for bilateral inguinal herniorrhaphy and circumcision two weeks prior to discharge.

On the day of surgery, the patient was $403 / 7$ weeks and measured $50.5 \mathrm{~cm}$ and $3.55 \mathrm{~kg}$. After a preoperative discussion with the surgical and anesthesia teams, the anesthetic plan was to proceed with a spinal and caudal epidural for the procedure. With a preexisting IV and an experienced pediatric surgeon, we were hopeful that the spinal anesthetic would provide sufficient anesthesia for the duration of the procedure as well as an awake caudal epidural at the conclusion of the procedure. Twenty minutes prior to transport from the Special Care Nursery to the operating room, EMLA 5\% cream was applied to the patient's lumbar spine. On arrival to the operating room, the patient was placed in the sitting position on a foam donut; standard ASA monitors and $2 \mathrm{~L} / \mathrm{min} \mathrm{O}_{2}$ via nasal cannula were applied. After the skin was prepped with betadine, $0.1 \mathrm{~mL}$ of lidocaine $1 \%$ was injected subcutaneously at the site of entry prior to midline insertion of a 1.5 inch, 22 gauge Quincke spinal needle at the L4-5 interspace. Correct placement was confirmed with CSF aspiration before injection of $0.8 \mathrm{~mL}$ of bupivacaine $0.5 \%$. He was immediately positioned supine and prepped and draped in usual fashion. To facilitate the surgery, his arms were immobilized with gauze ties and he was placated with a pacifier and oral sucrose solution. Surgical incision occurred ten minutes after the spinal anesthetic was administered. He remained comfortable and hemodynamically stable the entire time; vital assessments ranged from $96-100 \%\left(\mathrm{SpO}_{2}\right), 70-108 / 31-55 \mathrm{mmHg}$ (NIBP), 45-70 mmHg (MAP), 147-196 bpm (HR). Additionally, he remained on a D10W with electrolytes infusion at $100 \mathrm{~mL} / \mathrm{kg} /$ day during the procedure. He was transitioned to RA mid-procedure as his $\mathrm{SpO}_{2}$ did not require any oxygen supplementation. The surgical duration of bilateral inguinal herniorrhaphy and circumcision, from incision to closure, was forty-seven minutes. At the conclusion of the procedure, he was placed in the right lateral decubitus position for administration of acaudal anesthetic while fully awake. His skin was prepped with betadine prior to a single-shot administration of $6 \mathrm{~mL}$ of ropivacaine $0.1 \%+1: 200,000$ epinephrine with a 22 gauge standard angiocatheter in the caudal space; no blood or CSF was aspirated and test dose reaction was negative. After the caudal epidural anesthetic was administered, initial return of motor function of the lower extremities was noted. The patient tolerated the procedure well; blood loss was minimal. The patient was returned to the Special Care Nursery uneventfully where he continued to recover appropriately, with hemodynamic stability and $\mathrm{SpO}_{2}>94 \%$ on RA.

\section{Discussion}

Spinal anesthesia has had a notable history of safety and success in the pediatric anesthetic and surgical literature, with most of its interest revolved around formerly preterm or high-risk infants. It can be used as the sole anesthetic for a variety of infraumbilical and lower extremity procedures, often obviating the need for additional sedatives or anesthetics of which the effects on neurodevelopment are still presently unknown. Multiple studies have demonstrated that spinal anesthesia, in comparison to general anesthesia, can reduce postoperative apnea, oxygen desaturation, and bradycardia in formerly preterm or high-risk infants [1,2]. A 2015 Cochrane Database Review of seven small trials comparing spinal with general anesthesia in formerly preterm infants undergoing inguinal herniorrhaphy demonstrated a reduction in postoperative apnea risk by up to $47 \%$ in patients who received a spinal anesthetic without pre- or intraoperative sedatives compared to those who received a general anesthetic (typical RR 0.53, 95\% Cl 0.34-0.82; 4 studies; 129 infants). In fact, for every 4 infants who received a spinal anesthetic without additional sedation, 1 infant may be prevented from experiencing an episode of postoperative apnea $($ NNTB $=4)$ [2].

To serve as the sole anesthetic, spinal anesthesia must be administered successfully; this success often depends on the correct dosing of the local anesthetic among other factors. Unfortunately, high-level evidence is not presently available to guide the dosage of spinal LA used in children [8]. Additionally, a lot of dosing variation exists as well as method of dosing (either based on weight or age). For instance, while Verma, et al. and Suresh, et al. administered bupivacaine $0.5 \%$ at a dose of $0.5 \mathrm{mg} / \mathrm{kg}$ and $1 \mathrm{mg} / \mathrm{kg}$, respectively, Parthasarathy, et al. administered bupivacaine $0.5 \%$ at a dose of 
Citation: Tseng K, Liu CA (2020) Case Report: The Combined Spinal and Caudal (CSC) Anesthetic-Is this the New Ideal Anesthetic in a Formerly Preterm, High-Risk Infant with Chronic Lung Disease Undergoing Infraumbilical Surgery?. J Clin Anesth Pain Manag 4(2):131-133

age/5 (in $\mathrm{ml}$ ). Ultimately, we decided to dose our spinal with $1 \mathrm{mg} / \mathrm{kg}$ of bupivacaine $0.5 \%$ based on the large CSF volume in infants [10].

We report a successful awake spinal and caudal anesthetic in a formerly preterm, high-risk infant with chronic lung disease who underwent bilateral open inguinal herniorrhaphy and circumcision; we dosed our spinal with $1 \mathrm{mg} / \mathrm{kg}$ of bupivacaine $0.5 \%$ and also accounted for $0.1 \mathrm{ml}$ of dead space in the Quincke spinal needle. This dose provided sufficient anesthesia for the duration of the procedure as well as an awake caudal epidural, for postoperative pain control, at the conclusion of the procedure. By the time the caudal anesthetic was administered, the patient had regained motor function of his legs. No additional pre- or intraoperative sedative or anesthetic was required or administered (which avoided any sedation and its potential consequences such as apnea, bradycardia, or oxygen desaturation). The patient had an uneventful postoperative recovery: Vitals were monitored every 3 hours, and there were no episodes of apnea/bradypnea, hypotension, bradycardia, or oxygen desaturation in the first 24 hours, postoperatively. Additionally neonatal pain, sedation, and agitation scale (NPASS) was between 0 and 2 during this postoperative period. Our case demonstrates how a combined awake spinal and caudal anesthetic can be a beneficial anesthetic alternative, especially in a formerly preterm, highrisk infant with chronic lung disease who is at high risk for postoperative apnea. Moreover, it demonstrates spinal bupivacaine $0.5 \%$ can be safely and successfully performed at a dose of $1 \mathrm{mg} / \mathrm{kg}$ for a procedure lasting less than 1 hour.

\section{Funding Sources}

None.

\section{Conflicts of Interest}

None.

\section{References}

1. Krane E, Haberkem C, Jacobson LE (1995) Postoperative apnea, bradycardia, and oxygen desaturation in formerly premature infants: Prospective comparison of spinal and general anesthesia. Anesth Analg 80: 7-13.

2. Jones L, Craven PD, Lakkundi A, et al. (2015) Regional (spinal, epidural, caudal) versus general anesthesia in preterm infants undergoing inguinal herniorrhaphy in early infancy. Cochrane Database Syst Rev.

3. Frumiento C, Abajian JC, Vane DW (2000) Spinal anesthesia for preterm infants undergoing inguinal hernia repair. Ach Surg 135: 445-451.

4. Liu LM, Cote CJ, Goudsouzian NG, et al. (1983) Life-threatening apnea in infants recovering from anesthesia. Anesthesiology 59: 506-510.

5. Rappaport BA, Suresh S, Hertz S, et al. (2015) Anesthetic neurotoxicity--clinical implciations of animal models. N Engl J Med 372: 796-797.

6. Davidson AJ, Morton NS, Arnup SJ, et al. (2015) Apnea after awake regional and general anesthesia in infants: The general anesthesia compared to spinal anesthesia study--comparing apnea and neurodevelopmental outcomes, a randomized controlled trial. Anesthesiology 123: 38-54.

7. Whitaker EE, Wiemann BZ, DaJusta DG, et al. (2017) Spinal anesthesia for pediatric urological surgery: Reducing the theoretic neurotoxic effects of general anesthesia. J Pediatr Urol 13: 396-400.

8. Suresh S, Ecoffey C, Bosenberd A, et al. (2018) The European society of regional anaesthesia and pain therapy/American society of regional anesthesia and pain medicine recommendations on local anesthetics and adjuvants dosage in pediatric regional anesthesia. Reg Anesth Pain Med 43: 211-216.

9. Parthasarathy S, Senthilkumar T (2017) Age-based local anesthetic dosing in pediatric spinal anesthesia: Evaluation of a new formula - A pilot study in Indian patients. Anesth Essays Res 11: 627-629.

10. Verma D, Naithani U, Gokula C, et al. (2014) Spinal anesthesia in infants and children: A one year prospective audit. Anesth Essays Res 8: 324-329.

DOI: $10.36959 / 377 / 343$

Copyright: (c) 2020 Tseng K, et al. This is an open-access article distributed under the terms of the Creative Commons Attribution License, which permits unrestricted use, distribution, and reproduction in any medium, provided the original author and source are credited. 